ARTICLE

\title{
Radiative heat transfer exceeding the blackbody limit between macroscale planar surfaces separated by a nanosize vacuum gap
}

\author{
Michael P. Bernardi', Daniel Milovich ${ }^{1} \&$ Mathieu Francoeur ${ }^{1}$
}

Using Rytov's fluctuational electrodynamics framework, Polder and Van Hove predicted that radiative heat transfer between planar surfaces separated by a vacuum gap smaller than the thermal wavelength exceeds the blackbody limit due to tunnelling of evanescent modes. This finding has led to the conceptualization of systems capitalizing on evanescent modes such as thermophotovoltaic converters and thermal rectifiers. Their development is, however, limited by the lack of devices enabling radiative transfer between macroscale planar surfaces separated by a nanosize vacuum gap. Here we measure radiative heat transfer for large temperature differences $(\sim 120 \mathrm{~K})$ using a custom-fabricated device in which the gap separating two $5 \times 5 \mathrm{~mm}^{2}$ intrinsic silicon planar surfaces is modulated from 3,500 to $150 \mathrm{~nm}$. A substantial enhancement over the blackbody limit by a factor of 8.4 is reported for a 150-nm-thick gap. Our device paves the way for the establishment of novel evanescent wave-based systems.

\footnotetext{
${ }^{1}$ Radiative Energy Transfer Laboratory, Department of Mechanical Engineering, University of Utah, Salt Lake City, Utah 84112, USA. Correspondence and requests for materials should be addressed to M.F. (email: mfrancoeur@mech.utah.edu).
} 
$\mathrm{R}$ adiation heat transfer exceeding the blackbody limit at nanosize separation gaps has been experimentally confirmed in the scanning probe-surface ${ }^{1-3}$, scanning probe-film $^{4}$, microsphere-surface ${ }^{5-8}$, microsphere-film ${ }^{9}$ and microsphere-nanostructured surface ${ }^{10}$ configurations. Although the accuracy of fluctuational electrodynamics at sub-10 nm gaps has been questioned in the experiments of Kittel et al. ${ }^{2}$, the validity of this framework has been confirmed both experimentally ${ }^{3}$ and theoretically ${ }^{11}$ down to separation gaps of 2 and $1 \mathrm{~nm}$, respectively. Additional work involving micro/ nanostructures has also experimentally demonstrated the enhancement of thermal radiation in the near field ${ }^{12-16}$. The micro/nanosize surfaces involved in the aforementioned experiments, however, limit the amount of radiation that can be exchanged, such that these configurations cannot be readily applied to engineering systems such as thermophotovoltaic power generators ${ }^{17-26}$ and thermal rectifiers ${ }^{27-31}$. While the development of evanescent wave-based devices typically requires macroscale surfaces separated by a nanosize vacuum gap, experimental research on near-field radiative heat transfer between macroscale surfaces has mainly focused on relatively large, microsize separation gaps at cryogenic ${ }^{32,33}$ and room $^{34-37}$ temperatures. Recently, Ito et al. ${ }^{38}$ measured radiative heat transfer between millimetre-size fused quartz surfaces separated by pillars, also made of fused quartz, at a separation gap of $500 \mathrm{~nm}$. The results were twice that of fluctuational electrodynamics predictions because of excessive heat conduction through the pillars, which prevent the application of this configuration to engineering systems. Lim et al. ${ }^{39}$ measured a radiative heat transfer enhancement of 2.91 relative to the blackbody limit between two microstrips of doped silicon (Si) separated by a 400-nm-thick gap. Yet, significant heat transfer and radiation enhancement necessitate larger surfaces and a smaller separation gap, respectively.

The difficulty associated with maintaining a nanosize vacuum gap between macroscale planar surfaces is the main bottleneck, currently preventing the application of near-field thermal radiation to engineering systems ${ }^{15}$.

Here we address this bottleneck by measuring radiative heat transfer via a custom-fabricated device consisting of two planar $5 \times 5 \mathrm{~mm}^{2}$ intrinsic Si surfaces separated by a gap that can be modulated from $3,500 \mathrm{~nm}$ down to $150 \mathrm{~nm}$ via a compliant membrane and mechanical actuation. This device enables probing radiative heat transfer between macroscale surfaces for large temperature differences $(\Delta T \sim 120 \mathrm{~K})$ in multiple regimes, including those dominated by either propagating or evanescent modes. An excellent agreement between experimental results and fluctuational electrodynamics predictions ${ }^{40,41}$ is obtained, and a radiative transfer enhancement of 8.4 relative to the blackbody limit is measured for a separation gap of $150 \mathrm{~nm}$. The potential application of our device architecture to thermophotovoltaic power generation is also discussed.

\section{Results}

Experimental procedure. The near-field radiative heat transfer device, shown in Fig. 1, was manufactured using standard microfabrication techniques, as detailed in Supplementary Note 1 and Supplementary Fig. 1. The device consists of two $2.2 \times 2.2 \mathrm{~cm}^{2} \mathrm{Si}$ substrates separated by four rigid, $3.5-\mu \mathrm{m}$-tall SU-8 posts with a diameter of $250 \mu \mathrm{m}$. The bottom substrate was fabricated from a $525-\mu \mathrm{m}$-thick intrinsic Si wafer, while the top substrate was manufactured from a $521-\mu \mathrm{m}$-thick Si-on-insulator wafer with a $1-\mu \mathrm{m}$-thick buried silicon dioxide $\left(\mathrm{SiO}_{2}\right)$ layer. The surface roughness of the Si substrates was less than $1.2 \mathrm{~nm}$ as measured with a Zygo NewView optical profilometer. A 501- $\mu$ m-deep, 3.5-mm-wide trench was etched on the backside of the top Si substrate using deep-reactive ion etching and a buffered oxide etch solution. This resulted in a $20-\mu \mathrm{m}$-thick compliant $\mathrm{Si}$ membrane, allowing the $5 \times 5 \mathrm{~mm}^{2}$ emitter to move relative to the receiver under an applied force (the emitter-receiver portion of the device is identified by a dashed box in Fig. 1a,b). To avoid contact between the emitter and the receiver, four $\mathrm{SiO}_{2}$ stoppers with a diameter of $5 \mu \mathrm{m}$ and height of $150 \mathrm{~nm}$ were fabricated on the lower Si substrate, thus fixing the minimum separation gap $d$ to $150 \mathrm{~nm}$. The two Si substrates were precisely aligned and bonded in an EVG 520 IS wafer bonder. Testing of the device was conducted in a vacuum chamber $\left(P \approx 10^{-4} \mathrm{~Pa}\right)$ located in a class 1,000 clean room tent.

Heat transfer measurements were performed using the configuration shown in Fig. 1b,c. The temperature difference was maintained via thermoelectric (TE) modules (Custom Thermoelectric, 00701-9B30-22RU4) acting as a heat pump and cooler on the emitter and receiver sides, respectively. These TE modules enabled a maximum temperature difference of $\sim 120 \mathrm{~K}$ between the emitter and the receiver. The TE heat pump was mounted on a $500-\mu$ m-thick copper $(\mathrm{Cu})$ heat spreader located on the $5 \times 5 \mathrm{~mm}^{2} \mathrm{Si}$ emitter. The temperature of the outer surface of the emitter $T_{\mathrm{e}, \mathrm{o}}$ was measured via a thermistor (Selco, LSMC700A010KD002) embedded in the $\mathrm{Cu}$ heat spreader. The outer surface of the receiver was maintained at a temperature $T_{\mathrm{r}, \mathrm{o}}$ of $300 \mathrm{~K}$, monitored via a thermistor, by the TE cooler. The receiver and TE cooler were separated by a $500-\mu \mathrm{m}$-thick $\mathrm{Cu}$ heat spreader. The entire device was placed on a $\mathrm{Cu}$ heat sink mounted to the base of the vacuum chamber (see Fig. 1d) and all contact resistances were minimized using thermal grease (Arctic Silver Ceramique 2). Heat was supplied to the device at a rate $Q$, which is the sum of the heat rate into $\left(Q_{\text {in }}\right)$ and supplied by $\left(P_{\mathrm{HP}}\right)$ the TE heat pump. Since the device is in a vacuum, $Q_{\text {in }}$ is solely due to thermal emission by the stainless steel walls and aluminium door of the vacuum chamber near ambient temperature and is thus much smaller than $P_{\mathrm{HP}}$, such that $Q \approx P_{\mathrm{HP}}$. The heat supplied by the TE heat pump $Q$, partially spreading outside the emitter-receiver portion of the device, is divided into two contributions, namely radiation heat transfer at a separation gap $d$ between the emitter and receiver $Q_{\mathrm{e}-\mathrm{r}}$, and the background heat transfer $Q_{\text {back. }}$ The background heat rate $Q_{\text {back }}$ includes radiation outside the emitter-receiver portion of the device at a separation gap $\delta$, conduction through the SU-8 posts and conduction through the $\mathrm{SiO}_{2}$ stoppers when the device is in the closed position. Note that the thermal resistance associated with the separation gap (for example, $462.4 \mathrm{~K} \mathrm{~W}^{-1}$ for $d=150 \mathrm{~nm}$, $\left.T_{\mathrm{e}, \mathrm{o}}=420 \mathrm{~K}, T_{\mathrm{r}, \mathrm{o}}=300 \mathrm{~K}\right)$ is much larger than the thermal resistances of the emitter $\left(0.192 \mathrm{KW}^{-1}\right)$ and receiver $\left(0.162 \mathrm{~K} \mathrm{~W}^{-1}\right)$, such that the measured temperatures are approximately equal to temperatures of the inner surfaces of the emitter $\left(T_{\mathrm{e}}\right)$ and receiver $\left(T_{\mathrm{r}}\right)$, adjacent to the vacuum gap. The experimental procedure was validated by measuring conduction through a $1.1-\mathrm{mm}$-thick layer of borosilicate glass. In addition, using a technique similar to that of $\mathrm{Hu}$ et $a .^{35}$, radiation transfer was measured between $5 \times 5 \mathrm{~mm}^{2}$ planar $\mathrm{Si}$ surfaces separated by vacuum gaps of 500 and $200 \mathrm{~nm}$ maintained by low thermal conductivity $\left(0.18 \mathrm{~W} \mathrm{~m}^{-1} \mathrm{~K}^{-1}\right)$ polystyrene spherical particles. The validation procedure is detailed in Supplementary Note 2, while the conduction and radiation validation results are provided in Supplementary Figs 2 and 3, respectively.

Heat transfer measurements. The heat rate supplied by the TE heat pump as a function of the measured temperature difference $\Delta T$ between the emitter and receiver was compared against numerical predictions. Unprocessed experimental heat rates $Q$ that include radiation heat transfer between the emitter and receiver $Q_{\mathrm{e}-\mathrm{r}}$ as well as the background heat transfer $Q_{\text {back }}$ are 
a

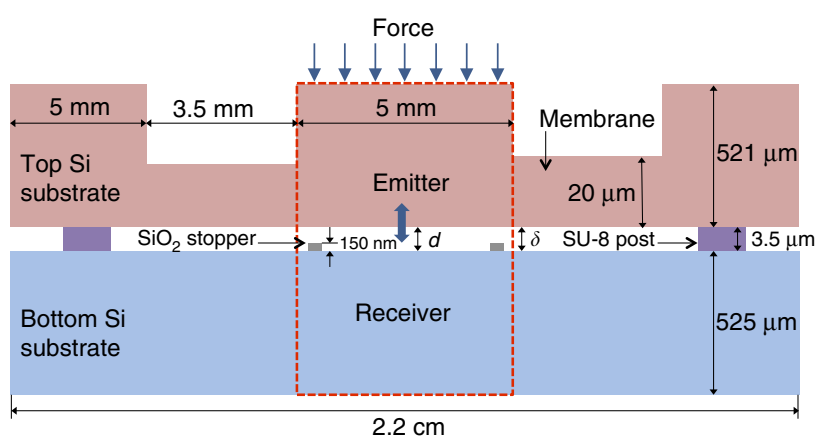

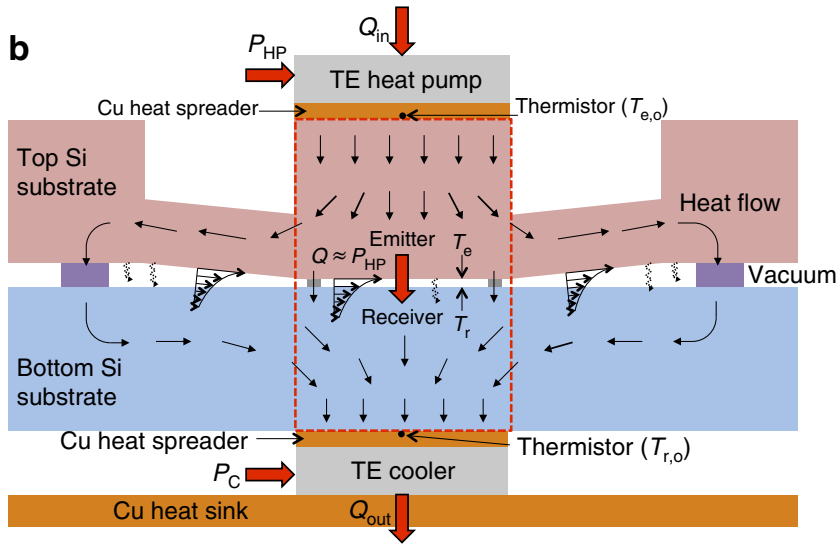

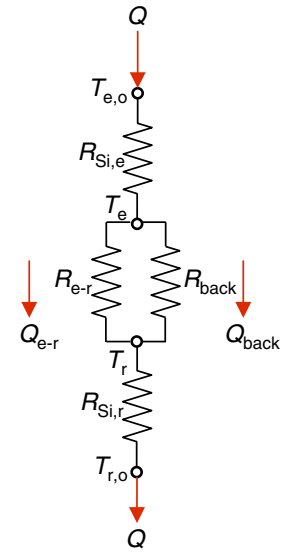

d

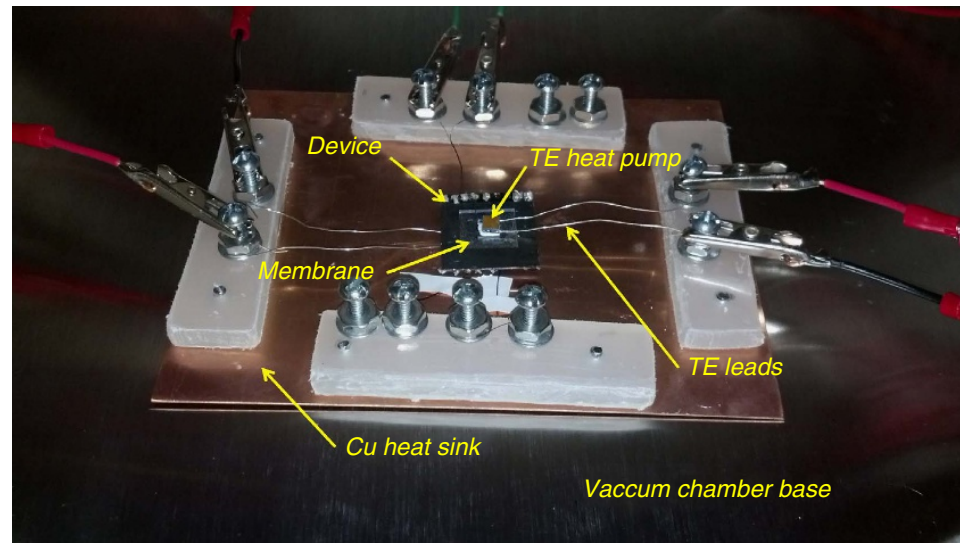

Figure 1 | Near-field radiative heat transfer device. (a) Device in the open position, where the emitter-receiver portion is identified by a dashed box. It consists of two silicon ( $\mathrm{Si}$ ) substrates separated by $3.5-\mu \mathrm{m}$-tall SU-8 posts. The gap between the emitter and the receiver, $d$, can be modulated by applying a force causing the membrane to flex. The minimum gap $d$ is $150 \mathrm{~nm}$ and corresponds to the height of the silicon dioxide $\left(\mathrm{SiO}_{2}\right)$ stoppers. The gap $d$ is uniform under an applied force, while the gap outside the emitter-receiver region, $\delta$, is non-uniform when a force is applied to the device. The $1-\mu m$-thick buried $\mathrm{SiO}_{2}$ layer in the top $\mathrm{Si}$ substrate is not shown. (b) Heat flow through the device in the closed position. The temperature difference is maintained by a TE heat pump and a TE cooler supplying power of $P_{\mathrm{HP}}$ and $P_{\mathrm{C}}$, respectively. $P_{\mathrm{HP}}$ is approximately equal to the heat rate through the device, $Q$, since the heat rate $Q_{\text {in }}$ due to thermal emission by the surroundings is negligible with respect to $P_{\mathrm{HP}}$. Heat transfer between the top and bottom Si substrates occurs via radiation within and outside the emitter-receiver region and by conduction through the $\mathrm{SU}-8$ posts and $\mathrm{SiO}_{2}$ stoppers when the device is in the closed position. The temperatures $T_{\mathrm{e}, \mathrm{o}}$ and $T_{\mathrm{r}, \mathrm{o}}$ are measured by thermistors and are approximately equal to $T_{\mathrm{e}}$ and $T_{\mathrm{r},}$ respectively. The device is mounted on a copper (Cu) heat sink dissipating heat, $Q_{\text {out, }}$ to the base of the vacuum chamber. (c) Equivalent thermal circuit of the device. $R_{\mathrm{Si}, \mathrm{e}}$ and $R_{\mathrm{Si}, \mathrm{r}}$ are the resistances of the Si emitter and receiver, $R_{\mathrm{e}-\mathrm{r}}$ is the resistance due to radiation between the emitter and receiver, $R_{\text {back }}$ is the resistance due to background heat transfer that includes radiation outside the emitter-receiver region as well as conduction through the SU-8 posts and $\mathrm{SiO}_{2}$ stoppers. The heat rate flowing through the device, $Q$, is the sum of heat rates due to radiation between the emitter and receiver, $Q_{e-r}$, and background heat transfer, $Q_{\text {back. }}$.

(d) Photograph of the near-field radiative heat transfer device in the vacuum chamber.

shown in Fig. 2a for temperature differences $\Delta T\left(=T_{\mathrm{e}}-T_{\mathrm{r}}\right)$ up to $120 \mathrm{~K}$. The separation gap $d$ between the emitter and receiver was modulated by using calibrated masses ranging from 0.9 to $5 \mathrm{~g}$, as detailed in Supplementary Note 3. The numerical predictions were obtained via a coupled fluctuational electrodynamicsCOMSOL Multiphysics comprehensive heat transfer model of the device taking into account radiation between the emitter and receiver, heat transfer by radiation outside the emitter-receiver region and conduction through the $\mathrm{SiO}_{2}$ stoppers and SU-8 posts. The details of the comprehensive model are provided in the Methods Section. Figure $2 \mathrm{~b}$ shows the temperature distribution in the device obtained from the model for a heat rate $Q$ of $0.92 \mathrm{~W}$ and a separation gap $d$ of $150 \mathrm{~nm}$. The agreement between experimental data and numerical predictions when the device is in the open $(d=3,500 \pm 22 \mathrm{~nm})$ and closed $(d=150 \pm 5 \mathrm{~nm})$ position is remarkable. The uncertainty associated with these gap sizes, identified as coloured bands in Fig. 2a, was determined experimentally by measuring the variation of the height of the
$\mathrm{SiO}_{2}$ and SU-8 layers used to create the stoppers and the posts, respectively. Using nominal gap values of 3,500 and $150 \mathrm{~nm}$ in the numerical simulations, a maximum relative difference between experiments and predictions of $9.1 \%$ is obtained for a 3,500-nmthick gap and $\Delta T=15.5 \mathrm{~K}$, while a minimum relative difference of less than $0.1 \%$ is achieved for a 150 -nm-thick gap and $\Delta T=84.2 \mathrm{~K}$. In addition, for all cases presented in Fig. 2a, radiation largely dominates heat transfer through the device. According to the model, the portion of the heat rate because of conduction reaches a maximum of $11.7 \%$ when the gap size is $3,500 \mathrm{~nm}$ and the temperature difference is $3.8 \mathrm{~K}$. For the case when the device is in the closed position $(d=150 \mathrm{~nm})$ and the temperature difference is $115.6 \mathrm{~K}$, the portion of the total heat rate due to conduction is at a minimum of $6.6 \%$. As explained in Supplementary Note 3 and Supplementary Fig. 4, it was not possible to determine exactly the intermediate gap sizes between the open and closed positions. As the force applied on the device was increased, it was observed that the heat rate $Q$ increased, 
a

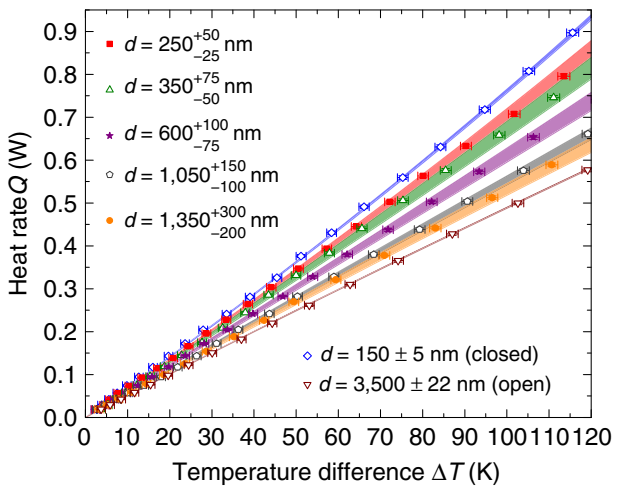

b

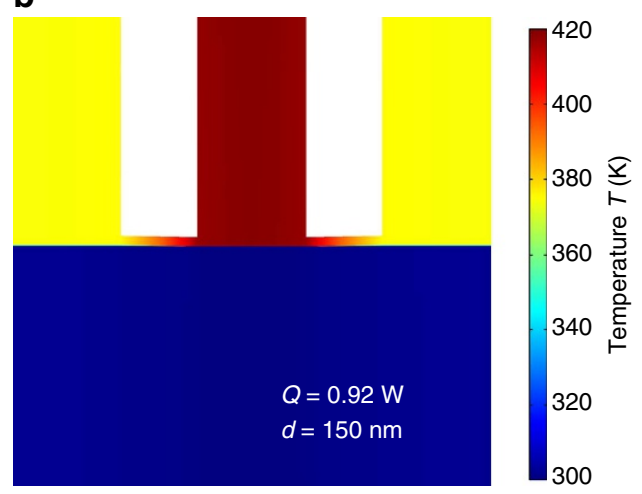

Figure 2 | Gap- and temperature-dependent heat rate. (a) Heat rate, $Q$, as a function of the temperature difference between the emitter and receiver, $\Delta T$, for various separation gaps, $d$. In all cases, the temperature of the receiver, $T_{r}$, is fixed at $300 \mathrm{~K}$. The symbols show unprocessed experimental data, while the coloured bands are numerical simulations obtained from the coupled fluctuational electrodynamics-COMSOL Multiphysics comprehensive model. The gap sizes $d$ in the open and closed positions are known, with some small uncertainty, from the manufacturing of the device and the associated measured heat rates are in good agreement with numerical predictions. It was not possible to measure directly the intermediate gap sizes, such that they were estimated from the comprehensive heat transfer model. (b) Simulated temperature distribution in the device via the comprehensive model for an input heat rate $Q$ of $0.92 \mathrm{~W}$, a separation gap $d$ of $150 \mathrm{~nm}$ and a fixed receiver temperature $T_{r}$ of $300 \mathrm{~K}$ resulting in an emitter temperature of $420 \mathrm{~K}$. Heat spreading outside the emitter portion of the device results in background heat transfer Qback.

because of a larger proportion of evanescent modes contributing to heat transfer, until the device was in the closed position. Intermediate gap sizes, shown in Fig. 2a, were estimated using the comprehensive model in combination with the measured heat rates and temperatures (see Methods section).

The radiative heat flux between the $5 \times 5 \mathrm{~mm}^{2}$ emitter and receiver without the background heat rate is shown in Fig. $3 \mathrm{a}$ as a function of the temperature difference. The agreement between experimental data and fluctuational electrodynamics predictions in the open and closed positions, for which the gap sizes were measured experimentally, is excellent. A maximum radiation transfer enhancement over the blackbody limit by a factor of 8.4 was measured for a gap size of $150 \mathrm{~nm}$ and a temperature difference of $115.6 \mathrm{~K}$. This constitutes the largest value recorded between two macroscale planar surfaces at non-cryogenic temperatures. The mechanism responsible for this enhancement can be understood by inspecting the dispersion relations shown in Fig. 3b, where the heat flux is plotted as a function of the angular frequency $\omega$ and parallel wavevector $k_{\rho}$ for gap sizes of $3,500,1,000,500$ and $150 \mathrm{~nm}$, and a temperature difference of $120 \mathrm{~K}$. Modes that are propagating in both $\mathrm{Si}$ and vacuum are contained within the region $k_{\rho}<k_{0}\left(=\omega / c_{0}\right)$, where $k_{0}$ is the magnitude of the wavevector in vacuum. Planck's theory of heat radiation solely accounts for these modes. Frustrated modes, propagating in $\mathrm{Si}$ and evanescent in vacuum, are characterized by parallel wavevectors $k_{0}<k_{\rho}<\operatorname{Re}(n) k_{0}$, where $n$ is the refractive index of Si. Surface modes are evanescent in both $\mathrm{Si}$ and vacuum and are described by $k_{\rho}>\operatorname{Re}(n) k_{0}$. The dispersion relations show clearly that the enhancement of radiative heat transfer is solely due to the additional contribution of frustrated modes in the near field, as intrinsic Si does not support surface modes such as surface phonon-polaritons or surface plasmon-polaritons. Here with a single device, we measured radiative heat transfer in various regimes, including those dominated by either propagating or evanescent modes. Indeed, although heat transfer via frustrated modes occurs at a gap of $3,500 \mathrm{~nm}$, their contribution is insufficient to exceed the blackbody predictions ( $\sim 68 \%$ of the heat flux is due to propagating modes). Conversely, radiation heat transfer is largely dominated by frustrated modes for a 150 -nm-thick gap and accounts for $\sim 88 \%$ of the heat flux between the emitter and the receiver.

\section{Discussion}

Near-field thermal radiation research is motivated by potential applications to energy conversion, heat flow management, imaging and micro/nanomanufacturing $17,21,31,42-47$. Among those, thermophotovoltaic power generation capitalizing on evanescent modes is a promising application of our device, as this technology requires both large surfaces and considerable near-field enhancement. For instance, when considering both the near-field enhancement and the size of the surfaces, our device provides a radiative heat rate more than one order of magnitude larger than in ref. 39. In addition, conductive heat losses in our device, negatively having an impact on thermophotovoltaic power generation $^{22,25,48}$, are minimized to $6.6 \%$ of the total heat rate when the near-field enhancement is maximum. For an emitter temperature of $420 \mathrm{~K}$, the photon energy at which thermal emission is maximum is $\sim 0.18 \mathrm{eV}$, such that our device could operate as a thermophotovoltaic power generator by replacing the receiver by a cell made of indium antimonide (InSb) having an absorption bandgap energy of $0.17 \mathrm{eV}$ (ref. 26). In the radiative limit $^{23}$, we estimated that the output power density and conversion efficiency of a thermophotovoltaic power generator made of intrinsic Si and InSb maintained at 420 and $300 \mathrm{~K}$, respectively, were $400 \mathrm{~W} \mathrm{~m}^{-2}$ and $2.9 \%$ for a 150 -nm-thick gap. This implies that a $5 \times 5 \mathrm{~mm}^{2}$ surface area would produce an electrical power of $\sim 10 \mathrm{~mW}$, a value that could be increased by using an array of devices. In comparison, a similar thermophotovoltaic system operating in the far-field regime with a blackbody source would lead to a significantly lower output power density of $33.4 \mathrm{~W} \mathrm{~m}^{-2}$ and the same conversion efficiency of $2.9 \%$. A thermophotovoltaic device operating with an emitter temperature of $420 \mathrm{~K}$ could potentially be used for recycling waste heat in electronic devices such as solar cells, where output power density is more important than conversion efficiency ${ }^{48}$. In an actual thermophotovoltaic power generator, one must also consider the impacts of electrical and thermal losses on system performance. This was done by Bernardi et al. ${ }^{25}$, where the results showed that, despite its broadband near-field enhancement, an emitter such as intrinsic Si supporting strictly propagating and frustrated modes is more beneficial to the performance of evanescent wave-based thermophotovoltaic power generators than a radiatively optimized emitter supporting surface modes. 
a

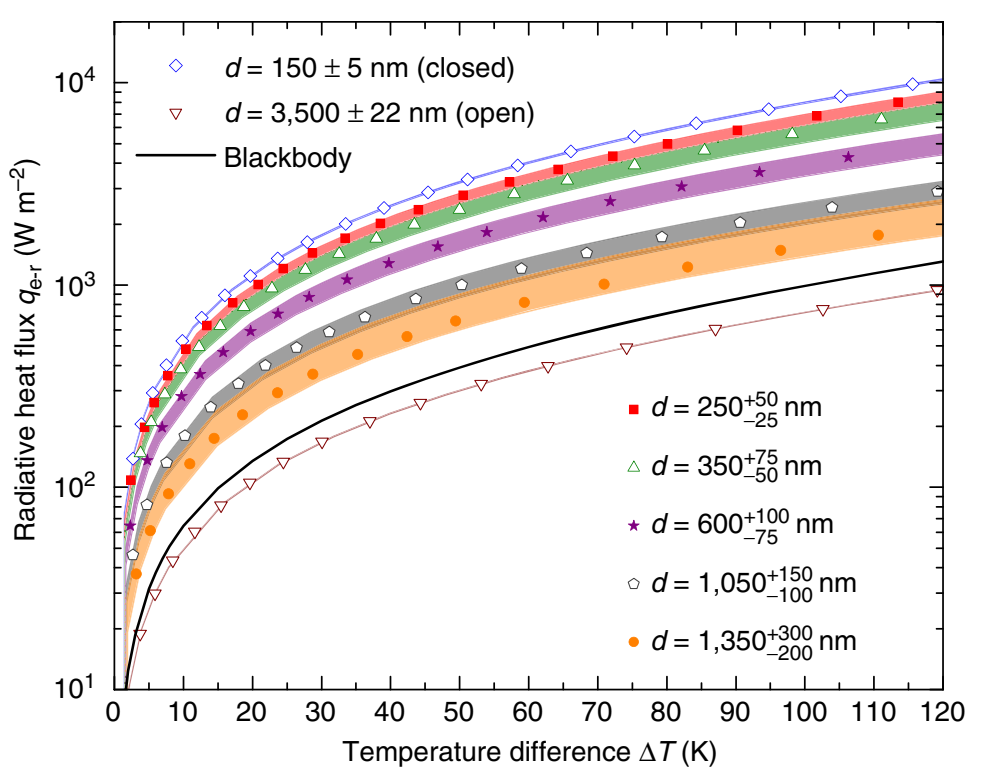

b
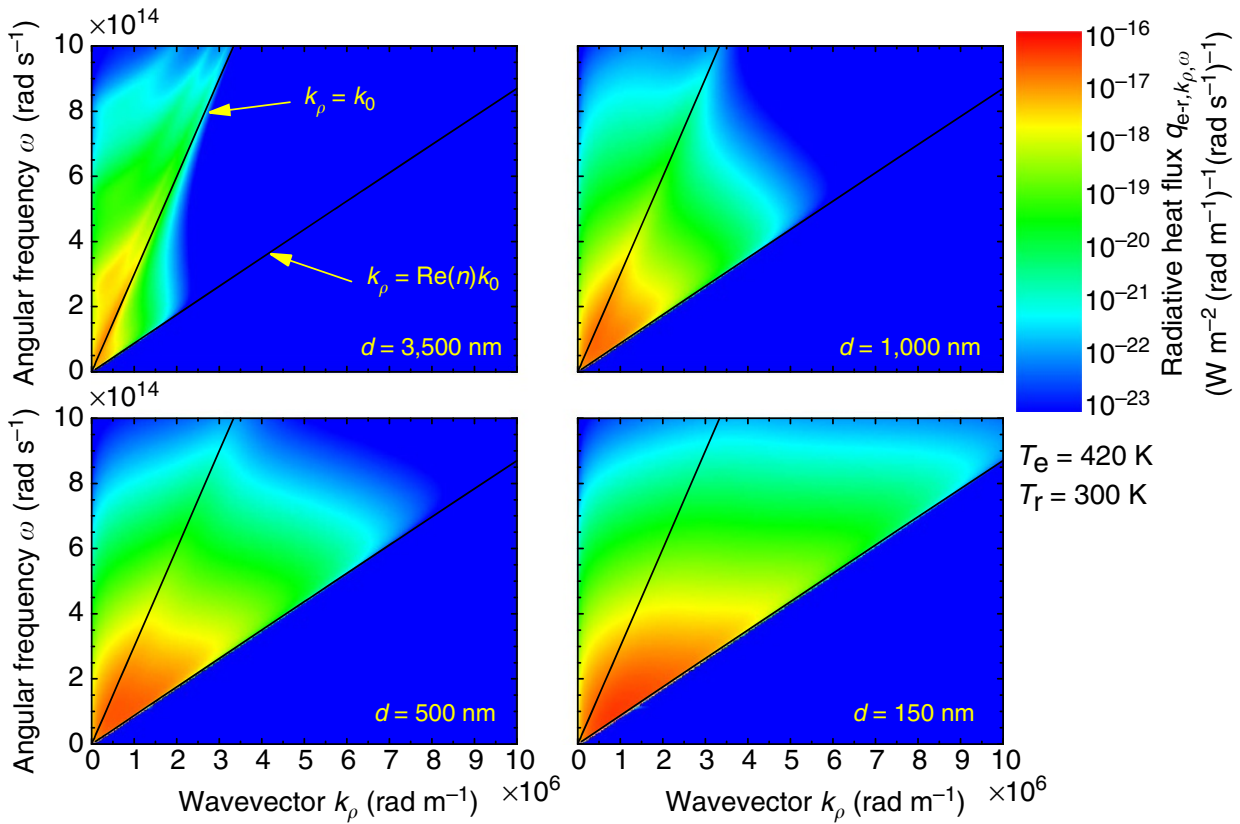

$T_{\mathrm{e}}=420 \mathrm{~K}$

$T_{\mathrm{r}}=300 \mathrm{~K}$

Figure 3 | Gap- and temperature-dependent radiative heat flux between the emitter and receiver. (a) The symbols show the experimental heat flux between the $5 \times 5 \mathrm{~mm}^{2}$ emitter and receiver, $q_{\mathrm{e}-r}$, where the background heat transfer has been subtracted. The coloured bands are fluctuational electrodynamics predictions. In the closed position $(d=150 \mathrm{~nm})$ and for a temperature difference $\Delta T$ of $115.6 \mathrm{~K}$, the experimental radiative heat flux exceeds the blackbody predictions by a factor of 8.4. In the open position $(d=3,500 \mathrm{~nm})$, the measured radiative heat flux is below the blackbody predictions due to a modest contribution from evanescent modes. (b) Calculated dispersion relations showing the radiative heat flux, $q_{\mathrm{e}-\mathrm{r}, k_{\rho}, \omega}$, per unit parallel wavevector $k_{\rho}$ and angular frequency $\omega$ for gaps of 3,500, 1,000, 500 and $150 \mathrm{~nm}$, and emitter $\left(T_{e}\right)$ and receiver $\left(T_{r}\right)$ temperatures of 420 and $300 \mathrm{~K}$, respectively. The region where $k_{\rho}$ is smaller than the magnitude of the wavevector in vacuum, $k_{0}$, corresponds to modes that are propagating in the vacuum gap. The zone defined by $k_{0}<k_{\rho}<\operatorname{Re}(n) k_{0}$, where $n$ is the refractive index of silicon ( $\mathrm{Si}$ ), describes frustrated modes that are propagating in Si but evanescent in the vacuum gap. Radiation enhancement in the near field for the case of intrinsic $\mathrm{Si}$ is solely due to these frustrated modes that have an increasing contribution to heat transfer as the separation gap $d$ decreases.

In summary, using a custom-fabricated device made of two $5 \times 5 \mathrm{~mm}^{2}$ intrinsic Si surfaces separated by a tunable vacuum gap, we measured a maximum radiation transfer enhancement over the blackbody limit by a factor of 8.4 for a 150 -nm-thick gap and a temperature difference of $115.6 \mathrm{~K}$ while minimizing heat conduction. Our near-field radiative heat transfer device, capable of delivering considerable radiation heat rates because of the large near-field enhancement and surface size, paves the way to the development of thermophotovoltaic systems converting evanescent modes into electrical power.

\section{Methods}

Experimental uncertainty analysis. The uncertainty associated with the experimental data is because of the temperature and heat rate measurements. For the temperature, the uncertainty stems from the ohmmeter (BK Precision, 889B) used to measure the resistance of the thermistors as well as the thermistors 
themselves. The error in the resistance measurement is given by the manufacturer specifications as $\pm(0.2 \%+0.1 \Omega)$ within the range from 100 to $1,000 \Omega$, and $\pm(0.1 \%+1.0 \Omega)$ for the range of $1-10 \mathrm{k} \Omega$. The uncertainty introduced by the thermistors is a function of temperature and the thermistors' change of resistance with temperature. A resistance reading of $9,225 \Omega$ corresponds to a temperature of $300 \pm 0.46 \mathrm{~K}$ while a resistance reading of $185.8 \Omega$ corresponds to a temperature of $420 \pm 0.85 \mathrm{~K}$. Combining the uncertainties introduced by the ohmmeter and the thermistors results in an overall uncertainty of $\pm 0.48 \mathrm{~K}$ at $300 \mathrm{~K}$ and $\pm 0.89 \mathrm{~K}$ at $420 \mathrm{~K}$.

The uncertainty associated with the heat rate is introduced by the power supply (BK Precision, 9121 A) connected to the TE heat pump. The uncertainty in the supplied current is $\pm(0.05 \%+2 \mathrm{~mA})$ and the uncertainty in the supplied voltage is $\pm(0.02 \%+3 \mathrm{mV})$. In addition, the resistance associated with the wires needs to be accounted for since it induces a small amount of power dissipation. A four-wire sensing technique was employed to account for the resistance of the wires leading up to the device. The resistance associated with the TE leads (see Fig. 1d) was measured to be $0.08 \pm 0.9 \mathrm{~m} \Omega$. For example, when $1.2 \mathrm{~A} \pm 2.6 \mathrm{~mA}$ and $0.84 \mathrm{~V} \pm 3.2 \mathrm{mV}$ is provided by the power supply $(1.01 \mathrm{~W} \pm 6.0 \mathrm{~mW})$, the power dissipated in the TE leads is $115.2 \pm 1.8 \mathrm{~mW}$. The power supplied to the TE heat pump, which is equivalent to the total heat rate, is then determined to be $894.8 \pm 7.8 \mathrm{~mW}$. These uncertainties are plotted as error bars in Fig. $2 \mathrm{a}$.

Computational model. Near-field radiative heat transfer was modelled using fluctuational electrodynamics ${ }^{40}$. The net radiative heat flux because of propagating and evanescent waves in the emitter-receiver portion of the device was calculated as follows ${ }^{41}$.

$$
q_{\mathrm{e}-\mathrm{r}}^{\text {prop }}=\frac{1}{4 \pi^{2}} \int_{0}^{\infty} \mathrm{d} \omega\left[\Theta\left(\omega, T_{\mathrm{e}}\right)-\Theta\left(\omega, T_{\mathrm{r}}\right)\right] \int_{0}^{k_{0}} \mathrm{~d} k_{\rho} k_{\rho} \sum_{\gamma=\mathrm{TE}, \mathrm{TM}} \frac{\left(1-\left|r_{01}^{\gamma}\right|^{2}\right)\left(1-\left|r_{02}^{\gamma}\right|^{2}\right)}{\left|1-r_{01}^{\gamma} r_{02}^{\gamma} e^{2 i R e\left(k_{20}\right) d}\right|^{2}}
$$

$q_{\mathrm{e}-\mathrm{r}}^{\mathrm{evan}}=\frac{1}{\pi^{2}} \int_{0}^{\infty} \mathrm{d} \omega\left[\Theta\left(\omega, T_{\mathrm{e}}\right)-\Theta\left(\omega, T_{\mathrm{r}}\right)\right] \int_{k_{0}}^{\infty} \mathrm{d} k_{\rho} k_{\rho} e^{-2 \operatorname{Im}\left(k_{\mathrm{z} 20}\right) d} \sum_{\gamma=\mathrm{TE}, \mathrm{TM}} \frac{\operatorname{Im}\left(r_{01}^{\gamma}\right) \operatorname{Im}\left(r_{02}^{\gamma}\right)}{\left|1-r_{01}^{\gamma} r_{02}^{\gamma} e^{-2 I m\left(k_{z 0}\right) d}\right|^{2}}$

where the subscripts 0,1 and 2, respectively, refer to the vacuum, the top $\mathrm{Si}$ substrate and bottom Si substrate, TE and TM refer to transverse electric and transverse magnetic polarizations, $\Theta(\omega, T)$ is the mean energy of an electromagnetic state calculated as $\hbar \omega /\left[\exp \left(\hbar \omega / k_{\mathrm{b}} T\right)-1\right], k_{\rho}$ and $k_{z}$ are the components of the wavevector parallel and perpendicular to the surface of the layers, while $r_{i j}^{\gamma}$ is the Fresnel reflection coefficient at the interface of media $i$ and $j$ in polarization state $\gamma$. Outside the emitter-receiver portion of the device, equations (1) and (2) were modified to account for the fact that the 20- $\mu \mathrm{m}$-thick membrane was optically thin. As such, the background-radiative heat flux due to propagating and evanescent waves was calculated as ${ }^{49}$ :

$$
\begin{aligned}
q_{\text {rad,back }}^{\text {prop }}= & \frac{1}{4 \pi^{2}} \int_{0}^{\infty} \mathrm{d} \omega\left[\Theta\left(\omega, T_{1}\right)-\Theta\left(\omega, T_{2}\right)\right] \\
& \int_{0}^{k_{0}} \mathrm{~d} k_{\rho} k_{\rho} \sum_{\gamma=\mathrm{TE}, \mathrm{TM}} \frac{\left(1-\left|R_{1}^{\gamma}\right|^{2}-\left|T_{1}^{\gamma}\right|^{2}\right)\left(1-\left|r_{02}^{\gamma}\right|^{2}\right)}{\left|1-R_{1}^{\gamma} r_{02}^{\gamma} e^{2 i R e\left(k_{20}\right) \delta}\right|^{2}} \\
q_{\text {rad,back }}^{\text {evan }}= & \frac{1}{\pi^{2}} \int_{0}^{\infty} \mathrm{d} \omega\left[\Theta\left(\omega, T_{1}\right)-\Theta\left(\omega, T_{2}\right)\right] \\
& \int_{k_{0}}^{\infty} \mathrm{d} k_{\rho} k_{\rho} e^{-2 \operatorname{Im}\left(k_{20}\right) \delta} \sum_{\gamma=\mathrm{TE}, \mathrm{TM}} \frac{\operatorname{Im}\left(R_{1}^{\gamma}\right) \operatorname{Im}\left(r_{0_{2}}^{\gamma}\right)}{\left|1-R_{1}^{\gamma} r_{02}^{\gamma} e^{-2 \operatorname{Im}\left(k_{z_{0}}\right) \delta}\right|^{2}}
\end{aligned}
$$

where $R_{1}^{\gamma}$ and $T_{1}^{\gamma}$ are the reflection and transmission coefficients of layer 1 in polarization state $\gamma$. These coefficients are calculated as $R_{1}^{\gamma}=\left[r_{01}^{\gamma}\left(1-e^{2 i k_{21} t}\right)\right] /$ $\left[1-\left(r_{01}^{\gamma}\right)^{2} e^{2 i k_{21} t}\right]$ and $T_{1}^{\gamma}=\left(t_{01}^{\gamma} t_{10}^{\gamma} e^{i k_{21} t}\right) /\left[1-\left(r_{01}^{\gamma}\right)^{2} e^{2 i k_{21} t}\right]$, where $t$ is the thickness of layer 1 , while $t_{i j}^{\gamma}$ is the Fresnel transmission coefficient at the interface of media $i$ and $j$ in polarization state $\gamma$. The net radiative heat flux used for producing the numerical results shown in Fig. 2a,b was obtained by summing equations (1) and (2) in the emitter-receiver region, and by summing equations (3) and (4) outside the emitter-receiver region. The net radiative heat flux in the emitter-receiver portion of the device shown in Fig. 3a was calculated by summing equations (1) and (2). The dispersion relations in Fig. 3b were generated by solving equations (1) and (2) per unit angular frequency $\omega$ and per unit parallel wavevector $k_{\rho}$. Note that the dielectric function of intrinsic $\mathrm{Si}$ was assumed to be independent of temperature in the range of operation and was obtained by curve-fitting the experimental data from ref. 50 .

Theoretical curves of heat rate $Q$ as a function of the temperature difference $\Delta T$ between the emitter and receiver for a specific separation gap $d$ were calculated using a coupled fluctuational electrodynamics-COMSOL Multiphysics comprehensive model to account for radiation transfer in the emitter-receiver region $Q_{\mathrm{e}-\mathrm{r}}$ as well as the background heat transfer $Q_{\text {back. }}$. Near-field radiative heat transfer was included in COMSOL by defining a fictional material, in place of the vacuum gap, characterized by a local, temperature-dependent effective thermal conductivity. In the emitter-receiver portion of the device, the effective thermal conductivity was calculated as $k_{\text {eff }}=q d / \Delta T$, where $q$ is the sum of equations (1) and (2). The effective thermal conductivity outside the emitter-receiver region was derived using $k_{\text {eff }}=q \delta / \Delta T$, where $q$ is the sum of equations (3) and (4), and by using the Derjaguin approximation ${ }^{7}$ to account for the variations of the separation distance $\delta$, assumed to be linear, when the device was not in the open position. Heat conduction through the $\mathrm{SU}-8$ posts and $\mathrm{SiO}_{2}$ stoppers was calculated using temperature-independent thermal conductivities of 0.2 and $1.3 \mathrm{~W} \mathrm{~m}^{-1} \mathrm{~K}^{-1}$ listed in refs 51,52 , respectively, while the temperature-dependent thermal conductivity provided in ref. 53 was used for intrinsic Si. A typical value for contact resistance of $2.5 \times 10^{-5} \mathrm{~K} \mathrm{~m}^{2} \mathrm{~W}^{-1}$ was imposed between the $\mathrm{Si}$ and $\mathrm{SiO}_{2}$ stoppers as well as between the Si and SU-8 posts ${ }^{54}$. For a given gap $d$ separating the emitter from the receiver, heat transfer simulations in the device were initiated by imposing the heat rate supplied by the TE heat pump $P_{\mathrm{HP}}(\approx Q)$, while the TE cooler was modelled as a constant temperature boundary condition $(300 \mathrm{~K})$ at the bottom face of the receiver. For these conditions, the temperature distribution in the device, and thus the temperature difference $\Delta T$ between the emitter and receiver, was determined using an iterative method where the effective thermal conductivity of the vacuum gap and the thermal conductivity of Si were calculated at the updated temperature. Iterative computations were repeated until a maximum absolute temperature difference between two successive iterations less than $0.001 \mathrm{~K}$ was obtained everywhere in the spatial grid. These simulations were repeated for a series of heat inputs $Q$ ranging from 0 to $1.4 \mathrm{~W}$ in increments of $0.04 \mathrm{~W}$, thus allowing the generation of a theoretical heat rate $Q$ as a function of the temperature difference $\Delta T$ between the emitter and receiver for a specific separation gap $d$. For instance, Fig. $2 \mathrm{~b}$ shows the temperature distribution in the device for a heat input $Q$ of $0.92 \mathrm{~W}$ and a separation gap $d$ of $150 \mathrm{~nm}$. For these conditions, the emitter reaches a uniform temperature of $420 \mathrm{~K}$, but heat is also dissipated outside the emitter region in the top Si substrate. It can also be seen that the bottom Si substrate has a nearly uniform temperature of $300 \mathrm{~K}$.

Validation of the coupled fluctuational electrodynamics-COMSOL Multiphysics model was performed by comparing numerical predictions against unprocessed experimental data measured when the device was in the open $(d=3,500 \pm 22 \mathrm{~nm})$ and closed $(d=150 \pm 5 \mathrm{~nm})$ positions. The uncertainty associated with these two gap sizes was used to calculate a theoretical band of heat rate $Q$ as a function of the temperature difference $\Delta T$. These bands, however, are fairly small because of the small uncertainty associated with these gap sizes, and are thus hardly visible in Fig. 2a.

Estimation of intermediate separation gap sizes. There was no direct method for measuring the separation gap between the emitter and receiver, except when the device was in the open and closed positions. Since the coupled fluctuational electrodynamics-COMSOL Multiphysics model was in excellent agreement with the measured heat rate as a function of the temperature difference in both the open and closed positions (see Fig. 2a), intermediate separation gap sizes were estimated using the aforementioned model. Specifically, a nominal gap size $d$ was determined by best fitting experimental data of nominal heat rate $Q$ versus temperature difference $\Delta T$ with numerical predictions. The uncertainty associated with the estimated gaps, shown in coloured bands in Figs $2 \mathrm{a}$ and 3a, was derived by best fitting experimental data of maximum $Q$ versus minimum $\Delta T$, and minimum $Q$ versus maximum $\Delta T$ with numerical predictions. These maximum and minimum values were determined from the uncertainty associated with the measured temperatures and heat rates.

Even if intermediate gap sizes were not determined from an independent measurement, the experimental results show clearly that heat transfer increases as the separation gap decreases because of an increasing contribution of evanescent modes. Heat transfer reaches saturation when the emitter comes into contact with the $\mathrm{SiO}_{2}$ stoppers. These observations are consistent with fluctuational electrodynamics predictions.

Data availability. The data that support the findings of this study are available from the corresponding author upon request.

\section{References}

1. Xu, J.-B., Läuger, K., Möller, R., Dransfield, K. \& Wilson, I. H. Heat transfer between two metallic surfaces at small distances. J. Appl. Phys. 76, 7209-7216 (1994).

2. Kittel, A. et al. Near-field heat transfer in a scanning thermal microscope. Phys. Rev. Lett. 95, 224301 (2005).

3. Kim, K. et al. Radiative heat transfer in the extreme near field. Nature 528, 387-391 (2015).

4. Worbes, L., Hellmann, D. \& Kittel, A. Enhanced near-field heat flow of a monolayer dielectric island. Phys. Rev. Lett. 110, 134302 (2013).

5. Narayanaswamy, A., Shen, S. \& Chen, G. Near-field radiative heat transfer between a sphere and a substrate. Phys. Rev. B 78, 115303 (2008). 
6. Shen, S., Narayanaswamy, A. \& Chen, G. Surface phonon polaritons mediated energy transfer between nanoscale gaps. Nano Lett. 9, 2909-2913 (2009).

7. Rousseau, E. et al. Radiative heat transfer at the nanoscale. Nat. Photon. 3, 514-517 (2009).

8. Shen, S., Mavrokefalos, A., Sambegoro, P. \& Chen, G. Nanoscale thermal radiation between two gold surfaces. Appl. Phys. Lett. 100, 233114 (2012).

9. Song, B. et al. Enhancement of near-field radiative heat transfer using polar dielectric thin films. Nat. Nanotechnol. 10, 253-258 (2015).

10. Shi, J., Liu, B., Li, P., Ng, L. Y. \& Shen, S. Near-field energy extraction with hyperbolic metamaterials. Nano Lett. 15, 1217-1221 (2015).

11. Chiloyan, V., Garg, J., Esfarjani, K. \& Chen, G. Transition from near-field thermal radiation to phonon heat conduction at sub-nanometre gaps. Nat. Commun. 6, 6755 (2015).

12. Guha, B., Otey, C., Poitras, C. B., Fan, S. \& Lipson, M. Near-field radiative cooling of nanostructures. Nano Lett. 12, 4546-4550 (2012).

13. Feng, C., Tang, Z., Yu, J. \& Sun, C. A MEMS device capable of measuring near-field thermal radiation between membranes. Sensors 13, 1998-2010 (2013).

14. St-Gelais, R., Guha, B., Zhu, L., Fan, S. \& Lipson, M. Demonstration of strong near-field radiative heat transfer between integrated nanostructures. Nano Lett. 14, 6971-6975 (2014).

15. St-Gelais, R., Zhu, L., Fan, S. \& Lipson, M. Near-field radiative heat transfer between parallel structures in the deep subwavelength regime. Nat. Nanotechnol. 11, 515-519 (2016).

16. Song, B. et al. Radiative heat conductances between dielectric and metallic parallel plates with nanoscale gaps. Nat. Nanotechnol. 11, 509-514 (2016).

17. DiMatteo, R. S. et al. Enhanced photogeneration of carriers in a semiconductor via coupling across a nonisothermal nanoscale vacuum gap. Appl. Phys. Lett. 79, 1894-1896 (2001).

18. Narayanaswamy, A. \& Chen, G. Surface modes for near field thermophotovoltaics. Appl. Phys. Lett. 82, 3544-3546 (2003).

19. Laroche, M., Carminati, R. \& Greffet, J.-J. Near-field thermophotovoltaic energy conversion. J. Appl. Phys. 100, 063704 (2006).

20. Park, K., Basu, S., King, W. P. \& Zhang, Z. M. Performance analysis of near-field thermophotovoltaic devices considering absorption distribution. J. Quant. Spectrosc. Radiat. Transfer 109, 305-316 (2008).

21. Basu, S., Zhang, Z. M. \& Fu, C. J. Review of near-field thermal radiation and its application to energy conversion. Int. J. Energ. Res. 33, 1202-1232 (2009).

22. Francoeur, M., Vaillon, R. \& Mengüç, M. P. Thermal impacts on the performance of nanoscale-gap thermophotovoltaic power generators. IEEE Trans. Energy Conv. 26, 686-698 (2011).

23. Ilic, O., Jablan, M., Joannopoulos, J. D., Celanovic, I. \& Soljacic, M. Overcoming the black body limit in plasmonic and graphene near-field thermophotovoltaic systems. Opt. Express 20, A366-A384 (2012).

24. Messina, R. \& Ben-Abdallah, P. Graphene-based photovoltaic cells for near-field thermal energy conversion. Sci. Rep. 3, 1383 (2013).

25. Bernardi et al. Impacts of propagating, frustrated and surface modes on radiative, electrical and thermal losses in nanoscale-gap thermophotovoltaic power generators. Sci. Rep. 5, 11626 (2015).

26. Lim, M., Jin, S., Lee, S. S. \& Lee, B. J. Graphene-assisted Si-InSb thermophotovoltaic system for low temperature applications. Opt. Express 23, A240-A253 (2015).

27. Otey, C. R., Lau, W. T. \& Fan, S. Thermal rectification through vacuum. Phys. Rev. Lett. 104, 154301 (2010).

28. Basu, S. \& Francoeur, M. Near-field radiative transfer based thermal rectification using doped silicon. Appl. Phys. Lett. 98, 113106 (2011).

29. Ben-Abdallah, P. \& Biehs, S.-A. Phase-change radiative thermal diode. Appl. Phys. Lett. 103, 191907 (2013).

30. Joulain, K., Ezzahri, Y., Drevillon, J., Rousseau, B. \& De Sousa Meneses, D. Radiative thermal rectification between $\mathrm{SiC}$ and $\mathrm{SiO}_{2}$. Opt. Express 23, A1388-A1397 (2015).

31. Ben-Abdallah, P. \& Biehs, S.-A. Contactless heat flux control with photonic devices. AIP Adv. 5, 053502 (2015).

32. Domoto, G. A., Boehm, R. F. \& Tien, C. L. Experimental investigation of radiative transfer between metallic surfaces at cryogenic temperatures. J. Heat Transfer 92, 412-416 (1970).

33. Kralik, T. et al. Strong near-field enhancement of radiative heat transfer between metallic surfaces. Phys. Rev. Lett. 109, 224302 (2012).

34. Hargreaves, C. M. Anomalous radiative transfer between closely-spaced bodies. Phys. Lett. 30A, 491-492 (1969).

35. Hu, L., Narayanaswamy, A., Chen, X. Y. \& Chen, G. Near-field thermal radiation between two closely spaced glass plates exceeding Planck's blackbody radiation law. Appl. Phys. Lett. 92, 133106 (2008).

36. Ottens, R. S. et al. Near-field radiative heat transfer between macroscopic planar surfaces. Phys. Rev. Lett. 107, 014301 (2011).

37. Ijiro, T. \& Yamada, N. Near-field radiative heat transfer between two parallel $\mathrm{SiO}_{2}$ plates with and without microcavities. Appl. Phys. Lett. 106, 023103 (2015).

38. Ito, K., Miura, A., Iizuka, H. \& Toshiyoshi, H. Parallel-plate submicron gap formed by micromachined low-density pillars for near-field radiative heat transfer. Appl. Phys. Lett. 106, 083504 (2015).
39. Lim, M., Lee, S. S. \& Lee, B. J. Near-field thermal radiation between doped silicon plates at nanoscale gaps. Phys. Rev. B 91, 195136 (2015).

40. Rytov, S. M., Kravtsov, Y. A. \& Tatarskii, V. I. Principles of Statistical Radiophysics 3: Elements of Random Fields. Ch. 3 (Springer, 1989).

41. Polder, D. \& Van Hove, M. Theory of radiative heat transfer between closely spaced bodies. Phys. Rev. B 4, 3303-3314 (1971).

42. Pendry, J. B. Radiative exchange of heat between nanostructures. J. Phys. Condens. Mater. 11, 6621-6633 (1999).

43. DeWilde, Y. et al. Thermal radiation scanning tunnelling microscopy. Nature 444, 740-743 (2006).

44. Babuty, A., Joulain, K., Chapuis, P.-O., Greffet, J.-J. \& DeWilde, Y. Blackbody spectrum revisited in the near field. Phys. Rev. Lett. 110, 146103 (2013).

45. Jones, A. C. \& Raschke, M. B. Thermal infrared near-field spectroscopy. Nano Lett. 12, 1475-1481 (2012).

46. O'Callahan, B. T., Lewis, W. E., Jones, A. C. \& Raschke, M. B. Spectral frustration and spatial coherence in thermal near-field spectroscopy. Phys. Rev. B 89, 245446 (2014).

47. Park, K. \& Zhang, Z. Fundamentals and applications of near-field radiative energy transfer. Front. Heat Mass Transfer 4, 013001 (2013).

48. DeSutter, J., Bernardi, M. P. \& Francoeur, M. Determination of thermal emission spectra maximizing thermophotovoltaic performance using a genetic algorithm. Energ. Convers. Manage 108, 429-438 (2016).

49. Francoeur, M., Mengüç, M. P. \& Vaillon, R. Spectral tuning of near-field radiative heat flux between two thin silicon carbide films. J. Phys. D Appl. Phys. 43, 075501 (2010).

50. Palik, E. D. Handbook of Optical Constants of Solids (Academic Press, 1998).

51. Chronis, N. \& Lee, L. P. Electrothermally activated SU-8 microgripper for single cell manipulation in solution. J. Microelectromech. Syst. 14, 857-863 (2005).

52. Hsu, T.-R. MEMS and Microsystems: Design, Manufacture, and Nanoscale Engineering. Ch. 7 (John Wiley and Sons, 2008).

53. Asheghi, M., Touzelbaev, M. N., Goodson, K. E., Leung, Y. K. \& Wong, S. S. Temperature dependent thermal conductivity of single-crystal silicon layers in SOI substrates. J. Heat Transfer 120, 30-36 (1998).

54. Bergman, T. L., Lavine, A. S., Incropera, F. P. \& DeWitt, D. P. in Fundamentals of Heat and Mass Transfer 7th edn Ch. 3 (John Wiley and Sons, 2011).

\section{Acknowledgements}

M.F. and M.P.B. acknowledge financial support from the National Science Foundation (Grant No. CBET-1253577). Financial support for D.M. was provided by the Undergraduate Research Opportunities Program (UROP) at the University of Utah. We also thank Rodolphe Vaillon for making a critical reading of this paper and John DeSutter for providing thermophotovoltaic performance predictions. This work was performed in part at the Utah Nanofab sponsored by the College of Engineering, Office of the Vice President for Research and the Utah Science Technology and Research (USTAR) initiative of the State of Utah. We appreciate the support of the staff and facilities that made this work possible.

\section{Author contributions}

This work was conceived by M.P.B. and M.F. Design, fabrication and testing of the device, as well as the associated numerical simulations, were performed by M.P.B. under the supervision of M.F. Validation of the experimental system via heat conduction and thermal radiation measurements was done by D.M. under the supervision of M.P.B. and M.F. The manuscript was written by M.P.B. and M.F. with comments from D.M.

\section{Additional information}

Supplementary Information accompanies this paper at http://www.nature.com/ naturecommunications

Competing financial interests: The authors declare no competing financial interests.

Reprints and permission information is available online at http://npg.nature.com/ reprintsandpermissions/

How to cite this article: Bernardi, M. P. et al. Radiative heat transfer exceeding the blackbody limit between macroscale planar surfaces separated by a nanosize vacuum gap. Nat. Commun. 7:12900 doi: 10.1038/ncomms12900 (2016).

This work is licensed under a Creative Commons Attribution 4.0 International License. The images or other third party material in this article are included in the article's Creative Commons license, unless indicated otherwise in the credit line; if the material is not included under the Creative Commons license, users will need to obtain permission from the license holder to reproduce the material. To view a copy of this license, visit http://creativecommons.org/licenses/by/4.0/ 